\title{
CONSTRAINTS ON THE PROGENITOR SYSTEM OF THE TYPE Ia SUPERNOVA 2014J FROM PRE-EXPLOSION HUBBLE SPACE TELESCOPE IMAGING
}

\author{
Patrick L. Kelly ${ }^{1}$, Ori D. Fox ${ }^{1}$, Alexei V. Filippenko ${ }^{1}$, S. Bradley Cenko ${ }^{2}$, Lisa Prato ${ }^{3}$, Gail Schaefer ${ }^{4}$, Ken J. Shen ${ }^{1,6}$, \\ WeiKang Zheng ${ }^{1}$, Melissa L. Graham ${ }^{1}$, and Brad E. Tucker ${ }^{1,5}$ \\ ${ }^{1}$ Department of Astronomy, University of California, Berkeley, CA 94720-3411, USA; pkelly@ astro.berkeley.edu \\ ${ }^{2}$ NASA/Goddard Space Flight Center, Code 662, Greenbelt, MD 20771, USA \\ ${ }^{3}$ Lowell Observatory, 1400 West Mars Hill Road, Flagstaff, AZ 86001, USA \\ ${ }^{4}$ The CHARA Array of Georgia State University, Mount Wilson Observatory, Mount Wilson, CA 91023, USA \\ ${ }^{5}$ Research School of Astronomy and Astrophysics, The Australian National University, Weston Creek, ACT 2611, Australia \\ Received 2014 March 17; accepted 2014 May 14; published 2014 June 26
}

\begin{abstract}
We constrain the properties of the progenitor system of the highly reddened Type Ia supernova (SN Ia) 2014J in Messier 82 (M82; $d \approx 3.5 \mathrm{Mpc}$ ). We determine the supernova (SN) location using Keck-II $K$-band adaptive optics images, and we find no evidence for flux from a progenitor system in pre-explosion near-ultraviolet through near-infrared Hubble Space Telescope (HST) images. Our upper limits exclude systems having a bright red giant companion, including symbiotic novae with luminosities comparable to that of RS Ophiuchi. While the flux constraints are also inconsistent with predictions for comparatively cool He-donor systems $(T \lesssim 35,000 \mathrm{~K})$, we cannot preclude a system similar to V445 Puppis. The progenitor constraints are robust across a wide range of $R_{V}$ and $A_{V}$ values, but significantly greater values than those inferred from the SN light curve and spectrum would yield proportionally brighter luminosity limits. The comparatively faint flux expected from a binary progenitor system consisting of white dwarf stars would not have been detected in the pre-explosion $H S T$ imaging. Infrared HST exposures yield more stringent constraints on the luminosities of very cool $(T<3000 \mathrm{~K})$ companion stars than was possible in the case of SN Ia $2011 \mathrm{fe.}$
\end{abstract}

Key words: binaries: symbiotic - supernovae: general - supernovae: individual (SN 2014J)

Online-only material: color figure

\section{INTRODUCTION}

The exceptional luminosity of Type Ia supernovae (SNe Ia), and the tight empirical relationships among the decline rate, color, and peak luminosity of their light curves (Phillips 1993; Riess et al. 1996), make SNe Ia useful probes of the cosmic expansion history (Riess et al. 1998; Perlmutter et al. 1999). SN Ia spectra and inferred ${ }^{56} \mathrm{Ni}$ masses (e.g., Mazzali et al. 2007) show reasonable agreement with models of the thermonuclear explosions of carbon-oxygen white dwarfs (Hillebrandt \& Niemeyer 2000; Kasen \& Plewa 2005; Kasen \& Woosley 2007; Kasen et al. 2009). Additional evidence for a comparatively old progenitor population comes from the presence of SNe Ia in passive galaxies, and the observation that they show no preference for the brightest regions of their hosts, in contrast to core-collapse explosions that also exhibit $\mathrm{H}$ - and He-deficient spectra (Kelly et al. 2008; see also Raskin et al. 2009).

For sufficiently nearby SNe Ia $(d \lesssim 10 \mathrm{Mpc})$, pre-explosion Hubble Space Telescope (HST) imaging has the sensitivity to detect several classes of candidate progenitor systems. Current constraints suggest that SN Ia progenitor systems consist primarily of either binary white dwarfs (Iben \& Tutukov 1984; Webbink 1984; Shen \& Bildsten 2014), or binaries where a single white dwarf accretes matter from a stellar companion (Whelan \& Iben 1973; Han \& Podsiadlowski 2004). For the latter, single-degenerate channel, the white dwarf gains matter from a companion star up to a point where its mass is close to the Chandrasekhar limit $\left(1.4 M_{\odot}\right)$, precipitating eventual thermonuclear runaway. Accretion onto a white dwarf primary can occur through Roche-lobe overflow from a secondary with a $\mathrm{H}$

\footnotetext{
6 Einstein Fellow.
}

envelope (van den Heuvel et al. 1992) or from a He star (Nomoto 1982; Yoon \& Langer 2003; Wang et al. 2009; Liu et al. 2010; Geier et al. 2013). Alternatively, in the case of the symbiotic channel, the white dwarf accretes mass from the wind generated by the secondary (Munari \& Renzini 1992; Patat et al. 2011). Characterizing the diversity of SN Ia progenitor systems may be useful for explaining evidence that the luminosities of $\mathrm{SNe}$ Ia have a $\sim 0.1 \mathrm{mag}$ dependence on the properties of the host galaxy, after correcting for light-curve shape and color (Kelly et al. 2010; Sullivan et al. 2010; Lampeitl et al. 2010; Childress et al. 2013).

While earlier analyses have found useful nondetections at SN Ia explosion sites (e.g., Maoz \& Mannucci 2008; Nelemans et al. 2008), Li et al. (2011) were able to place significantly fainter limits on the luminosity at the explosion site of SN Ia 2011fe in M101 ( $d \approx 6.4$ Mpc; Shappee \& Stanek 2011). Their nondetection excludes a bright red giant companion, specifically both model Galactic progenitor symbiotic systems RS Ophiuchi (RS Oph) and T Coronae Borealis (T CrB), as well as the He-star system V445 Puppis (V445 Pup).

Here we report constraints on the progenitor system of SN 2014J using pre-explosion near-ultraviolet (UV) through near-infrared (IR) HST imaging of the explosion site whose coordinates we measure using Keck-II adaptive optics (AO) imaging. Section 2 provides a brief summary of the discovery and early analysis of the spectra and light curve of SN 2014J. In Section 3, we describe the Keck AO and HST pre-explosion images that we analyze in this paper. The methods we use to extract upper luminosity limits for the progenitor system are explained in Section 4, while Section 5 presents constraints on possible progenitor systems. Section 6 provides a summary of our conclusions. 


\section{DISCOVERY AND EARLY ANALYSIS OF SN 2014J}

SN 2014J was discovered by Fossey et al. (2014) on 2014 January 21 (UT dates are used throughout this paper) during a University College London class observing session. Zheng et al. (2014) found that the light curve favors a time of first light of January 14.75 ( \pm 0.21 days), and showed that the early evolution of the light curve can be best described by a varying power law that shows a more rapid initial rise in luminosity than a $t^{2}$ dependence.

Tendulkar et al. (2014; also Goobar et al. 2014) acquired $K$-band images with the Near Infrared Camera 2 (NIRC2) in conjunction with the AO system (Wizinowich et al. 2006) on the Keck-II 10 m telescope. The corrected point-spread function (PSF) of their coadded image had a full width at half-maximum intensity (FWHM) of 0'.36 (measured at a $10^{\prime \prime}$ offset from SN $2014 J)$. Goobar et al. (2014) consider a source $0.2(5 \sigma)$ from the measured SN position as a potential companion, although they suggest that a radio upper limit (Chandler \& Marvil 2014) disfavors this possibility by setting a prohibitively low constraint on a donor star's mass-loss rate.

After removing $E(B-V)_{\mathrm{MW}}=0.14$ mag Galactic reddening determined by Schlafly \& Finkbeiner (2011), Goobar et al. (2014) find that the shape of the optical SN 2014J spectrum may be reproduced by applying a low $R_{V}=A_{V} / E(B-V)$ extinction curve to a spectrum of SN $2011 \mathrm{fe}$, a spectroscopically normal and unreddened SNe Ia; a Cardelli et al. (1989) reddening law with $E(B-V)_{\mathrm{SN}}=1.22 \pm 0.05 \mathrm{mag}$ and $R_{V}=1.40 \pm 0.15$ provides the best match to the observed SN 2014J spectrum. The favored value of $A_{V}$ is consistent with the measured equivalent width of the diffuse interstellar band at $5780 \AA$.

Nielsen et al. (2014) analyze archival observations taken with the Chandra X-ray telescope of the position of SN 2014J. They are not able to exclude a low-temperature $\left(k T_{\text {eff }} \lesssim\right.$ $80 \mathrm{eV}$ ) supersoft X-ray source coincident with the explosion. Additionally, they find that the explosion coordinates lie near the center of a $\sim 200 \mathrm{pc}$ structure of diffuse X-ray emission with inferred mass $\sim 3 \times 10^{4} M_{\odot}$, which they suggest may be a bubble inflated by one or more previous supernovae (SNe). Nielsen et al. (2014) consider the possibility that SN 2014J may be associated with a prompt channel linked to nearby recent star formation.

\section{DATA}

\subsection{Keck-II NIRC2 Adaptive Optics Imaging}

To locate the SN site in pre-explosion HST exposures with high precision, we acquired wide-field NIRC2 $K$-band AO images of the site of SN 2014J on 2014 January 25. The SN was used as the guide star to measure tip-tilt corrections. The FWHM natural seeing produced a PSF with 0.'65 FWHM, while the AO-corrected images have a PSF FWHM of $0^{\prime \prime} 1$ near the SN position.

We first subtracted the median of a stack of bias exposures and then applied flat-field and distortion ${ }^{7}$ corrections to the NIRC2 images. Although exposures of SN 2014J were acquired for the minimum possible integration time, the number of counts registered by the several pixels closest to the peak of the PSF exceeded the range within which the detector has a linear response. Both to register the $18 \mathrm{AO}$ exposures and then to measure the coordinates of the PSF of SN 2014J, we needed to determine the center of the PSF of the SN. We therefore fit a two-dimensional Gaussian model to the SN pixel intensity

\footnotetext{
https://www2.keck.hawaii.edu/inst/nirc2/dewarp.html
}

distribution in each of the 18 exposures and allowed the PSF center coordinates, FWHM, and normalization to vary.

Saturated pixels in the NIRC2 detector assume low data number (DN) values during readout. For each exposure, we identified (through visual inspection) and masked the one or two pixels whose low DN values were consistent with a saturated pixel. Our $\chi^{2}$ goodness-of-fit statistic excludes pixels where the model value was in excess of the nonlinearity threshold. Centers of the fitted Gaussian models coincided with the positions of the most saturated pixel. The NIRC2 exposures show no evidence for bleeding from saturated pixels.

We then used the best-fitting SN coordinates to align the reduced images. The positions of the intensity peaks of many sources in M82 show variation with wavelength; thus, to mitigate the contribution of this astrometric noise and to maximize the number of common sources, we registered the coadded $K$-band image against archival HST F160W $(H)$ images. To minimize the effect of any remaining astrometric distortion in the NIRC2 coadded image, we used sources only within the central $16^{\prime \prime} \times 16^{\prime \prime}$ section of the NIRC2 coadded image when cross-registering with the HST F160W image. After extracting sources with SExtractor (Bertin \& Arnouts 1996) and excluding extended objects with $r_{50}>3$ pixels, we used the tweakreg routine in the astrodrizzle package ${ }^{8}$ to fit for an astrometric solution from 68 common objects.

Figure 1 shows the astrometrically matched, coadded $K$-band exposure obtained with the NIRC2 AO system, along with the coadded $H S T$ F160W image. The position of the SN is $\alpha=9^{\mathrm{h}} 55^{\mathrm{m}} 42.137(9)^{\mathrm{s}}, \delta=+69^{\circ} 40^{\prime} 25.40(5)^{\prime \prime}(\mathrm{J} 2000.0)$ in the World Coordinate System (WCS) of the drizzled F160W image 9 available from the Hubble Legacy Archive (HLA). ${ }^{10}$ The rms of the offsets between pairs of matched objects after applying the astrometric solution is 0.057 in R.A. and 0.'055 in decl.

\subsection{Pre-explosion HST Imaging}

Table 1 provides a list of the HST imaging data sets of the explosion site that we use to place limits on the flux of the progenitor. Except for the Advanced Camera for Surveys (ACS) exposures, the images within each data set were processed, drizzled, and coadded to a common pixel grid by the HLA team. The ACS coadded mosaics of drizzled images that we analyze are High Level Science Products made available by the observers who acquired the data (Proposal 10776; PI: M. Mountain).

\section{METHODS}

We find an astrometric solution that aligns each coadded, drizzled $H S T$ image (see Table 1) with the F160W reference coadded image through a sequence of two steps. The objective is to achieve an accurate astrometric solution near the coordinates of SN 2014J, so we trim each HST image to a $30^{\prime \prime} \times 30^{\prime \prime}$ subsection centered on the $\mathrm{SN}$ position. In the first step, we use the $\mathrm{IRAF}^{11} \mathrm{ccmap}$ routine to compute an approximate astrometric alignment using 5-10 sources in common between the images. Next we use Source Extractor (SExtractor; Bertin \& Arnouts 1996) to extract a catalog of objects in each image, and we derive a final astrometric solution from $\sim 70-500$ sources using the tweakreg routine in the astrodrizzle package.

In Figure 2, we show our measured SN coordinates, as well as those obtained by Tendulkar et al. (2014), in a coadded image

\footnotetext{
8 http://drizzlepac.stsci.edu/

9 hst_11360_r9_wfc3_ir_f160w_drz.fits

10 http://hla.stsci.edu/

11 http://iraf.noao.edu/
} 

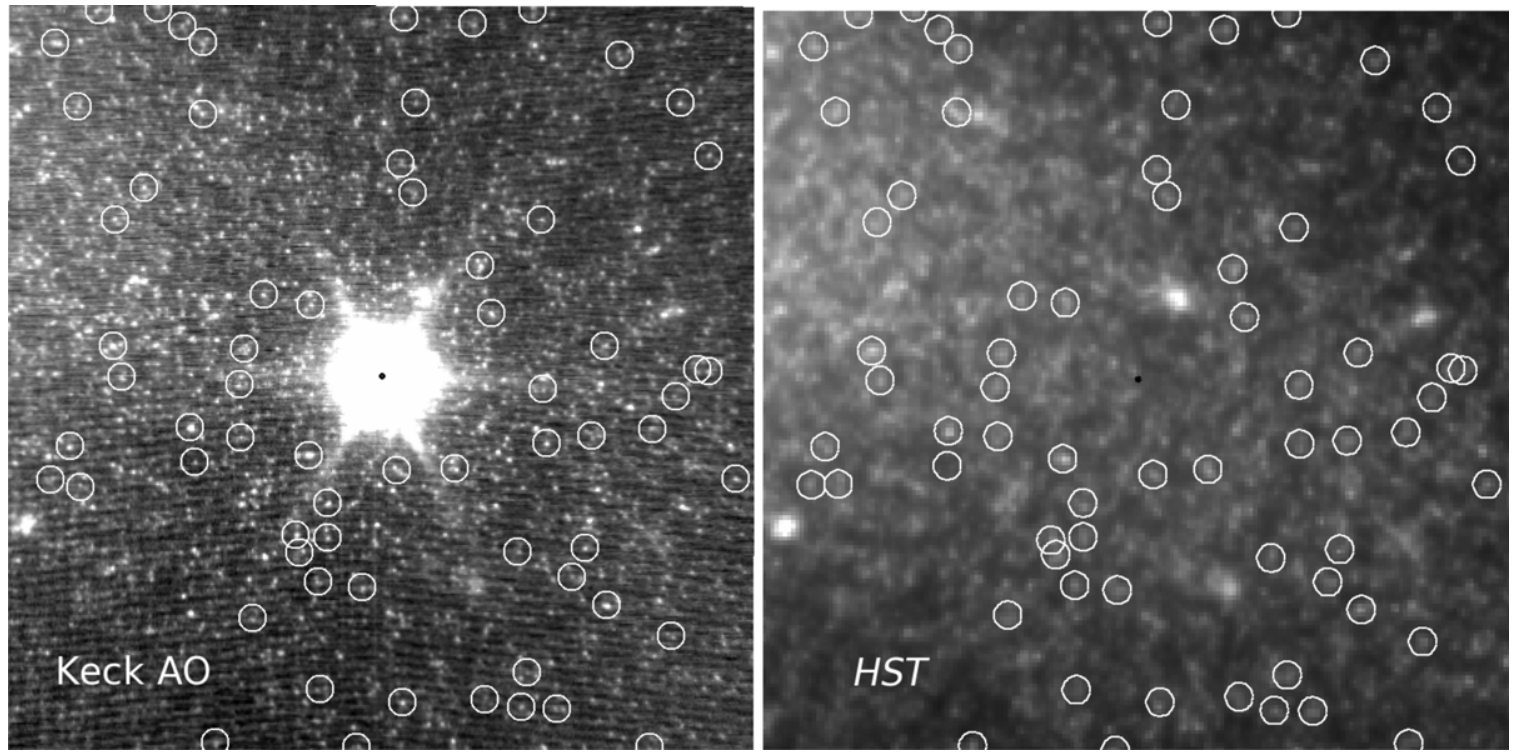

Figure 1. Coadded Keck-II K-band NIRC2 AO (left) and HST pre-explosion F160W (right) exposures of the location of SN 2014J. We use only the central 16" $\times 16^{\prime \prime}$ of the distortion-corrected AO image to perform astrometric registration. The 68 sources used for registration are identified with white circles, while the position of SN 2014J is marked by a black circle with radius corresponding to the uncertainty in that position estimate.

Table 1

HST Data Sets and Upper Absolute Magnitude Limits on Point-source Flux at Explosion Site

\begin{tabular}{|c|c|c|c|c|c|c|c|}
\hline Instrument & Aperture & Filter & UT Date Obs. & Exp. Time (s) & Prop. No. & Visual Limit & $3 \sigma$ Background Limit \\
\hline WFC3 & UVIS & F225W & 2010-01-01 & 1665.0 & 11360 & 26.50 & 26.80 \\
\hline WFC3 & UVIS & F336W & 2010-01-01 & 1620.0 & 11360 & 26.71 & 27.23 \\
\hline ACS & WFC & $\mathrm{F} 435 \mathrm{~W}$ & 2006-09-29 & 1800.0 & 10766 & 26.30 & 27.05 \\
\hline WFC3 & UVIS & F487N & $2009-11-17$ & 2455.0 & 11360 & 26.01 & 25.94 \\
\hline WFC3 & UVIS & F502N & 2009-11-17 & 2465.0 & 11360 & 25.93 & 26.28 \\
\hline WFPC2 & WF & F502N & $1998-08-28$ & 3600.0 & 6826 & 21.76 & 22.70 \\
\hline WFC3 & UVIS & F547M & 2010-01-01 & 1070.0 & 11360 & 26.14 & 25.94 \\
\hline WFPC2 & WF & F547M & $1998-08-28$ & 100.0 & 6826 & 21.63 & 22.12 \\
\hline $\mathrm{ACS}$ & WFC & F555W & $2006-03-29$ & 1360.0 & 10766 & 26.42 & 26.52 \\
\hline WFPC2 & WF & F631N & $1998-08-28$ & 1200.0 & 6826 & 21.43 & 22.17 \\
\hline ACS & WFC & F658N & 2004-02-09 & 700.0 & 9788 & 24.63 & 24.76 \\
\hline ACS & WFC & F658N & $2006-03-29$ & 4440.0 & 10766 & 25.06 & 25.17 \\
\hline WFPC2 & WF & F658N & $1997-03-16$ & 1200.0 & 6826 & 21.31 & 21.86 \\
\hline WFC3 & UVIS & F673N & $2009-11-15$ & 2760.0 & 11360 & 24.53 & 25.62 \\
\hline ACS & WFC & F814W & $2006-03-29$ & 700.0 & 10766 & 24.83 & 25.09 \\
\hline WFC3 & IR & F110W & $2010-01-01$ & 1195.39 & 11360 & 23.54 & 23.51 \\
\hline WFC3 & IR & $\mathrm{F} 128 \mathrm{~N}$ & $2009-11-17$ & 1197.69 & 11360 & 22.90 & 22.85 \\
\hline WFC3 & IR & F160W & 2010-01-01 & 2395.39 & 11360 & 22.43 & 22.48 \\
\hline WFC3 & IR & F164N & $2009-11-17$ & 2397.7 & 11360 & 21.98 & 22.17 \\
\hline
\end{tabular}

Notes. Limiting magnitudes in the Vega system for point sources near the explosion coordinates in the HST images. Visual limiting magnitudes are estimated by injecting a point source of increasing brightness in close proximity to the AO explosion coordinates, and identifying when a source is clearly detected. The $3 \sigma$ background detections are computed using the rms of the background measured in a region without point sources or pronounced background gradients.

of all pre-explosion $H S T$ exposures, and the F435W (Johnson $B$ ), F814W (Wide $I$ ), and F160W $(H) H S T$ coadded images. The Tendulkar et al. (2014) position was reported relative to the WCS of the HLA F814W image, and we use our astrometric registration of the images to determine the location of the Tendulkar et al. (2014) position in our reference F160W image. The SN 2014J position that we measure is offset by $0{ }^{\prime} 08$ from the coordinates we calculate for the Tendulkar et al. (2014) F814W position in the F160W image.

The angular distance between the position we estimate and the preliminary coordinates reported by Tendulkar et al. (2014) may arise from several differences between our AO coadded images and astrometric fitting. These include the substantially improved resolution of our NIRC2 AO exposures ( $\left.0^{\prime \prime} 1\right)$ compared to those analyzed by Tendulkar et al. (2014; 0'36), our restriction of cross-matched sources to those inside of the central $16^{\prime \prime} \times 16^{\prime \prime}$ region of the $40^{\prime \prime} \times 40^{\prime \prime}$ wide-field NIRC2 camera to minimize the effects of residual distortion, the numbers of matched sources ( 68 and 8 , respectively) incorporated into the astrometric fit by the two analyses, and our matching of sources in the $K$-band NIRC2 image against the near-IR HST F160W image as opposed to the $I$-band F $814 \mathrm{~W}$ image to be able to minimize source confusion and the effects of differential reddening.

\subsection{Upper Flux Limits}

As may be seen in the representative images in Figure 2, the local environment of SN 2014J exhibits strong surface brightness variations from both resolved and unresolved sources, as 

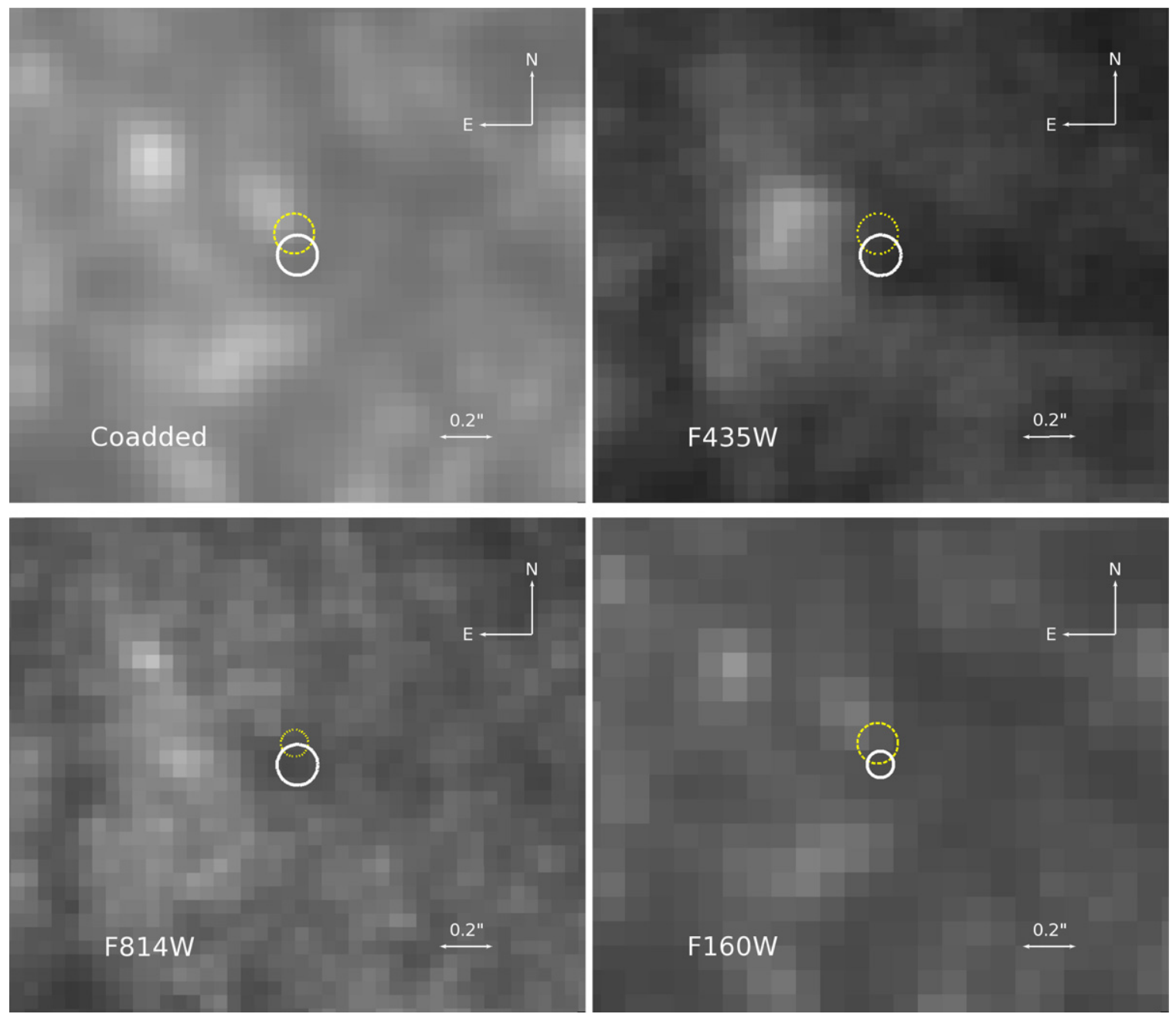

Figure 2. AO position of SN 2014J in a coadded image of all pre-explosion $H S T$ exposures, as well as in coadded F435W, F814W, and F160W HST images. The center of the solid white circle shows the position that we measure from our AO data, while the center of the dashed yellow circle corresponds to the position published by Tendulkar et al. (2014; also Goobar et al. 2014) from separate AO observations. The root-mean square (rms) scatter of the astrometric fit between our NIRC2 AO image and the HLA F160W image is 0'.057 in R.A. and 0'”055 in decl., and that reported by Tendulkar et al. (2014) relative to the HLA F814W image is 0'.023 in R.A. and $0^{\prime \prime} 042$ in decl. The radii of the circles shown in each image correspond to the positional uncertainty of the SN in either the F160W or F814W image, respectively, convolved when appropriate with the rms astrometric scatter between images in different bands (e.g., F160W and F435W). Our position is farther from a source considered as a possible progenitor candidate by Goobar et al. (2014) and is coincident with a region having strong extinction from dust.

(A color version of this figure is available in the online journal.)

well as strong and varying extinction by dust. As in Maoz \& Mannucci (2008), our approach for estimating nondetection limits is to add artificial point sources. We create a sequence of images, each with a superimposed artificial point source having FWHM appropriate to the $H S T$ instrument and bandpass filter. We create a pixelated Gaussian PSF and use the PyFITS package $^{12}$ to add the artificial source to the HST image. We place the PSF $\sim 1-2$ pixels in a randomly determined direction away from the $\mathrm{SN}$ explosion site to avoid superimposing the PSF on any possible underlying counts from a progenitor, which would yield a biased upper limit. These offset positions are sufficiently modest that the local background is comparable to that at the $\mathrm{SN}$ site.

The artificial point source in each successive image contains 0.1 mag additional flux than that in the previous image. We visually determine the limiting magnitude by identifying the first image for which the brightest pixel of the injected source has more counts than the neighboring local maxima produced by photon shot noise and background variation, and where pixels

\footnotetext{
12 http://www.stsci.edu/institute/software_hardware/pyfits
}

adjacent to the peak of the injected source have elevated counts consistent with a PSF.

To estimate the statistical significance of these visual detections for use in likelihood computations, we compute detection limits using a complementary technique. We calculate the rms of the background flux in a region without detected sources or a strong intensity gradient. We consider a $3 \sigma$ detection to be one for which the source flux exceeds the background rms inside a 6 pixel aperture by a factor of three.

A circular aperture enclosing $80 \%$ of the flux of a point source is the default choice for most HST instruments when computing the signal-to-noise ratio $(\mathrm{S} / \mathrm{N})$ of a flux measurement using the Exposure Time Calculator. ${ }^{13}$ For the ACS Wide Field Camera (WFC), this corresponds to an area that includes $\sim 44$ pixels (and corresponding background noise), and yields a limiting magnitude that is $\sim 0.7$ mag brighter and less sensitive than the value we compute for a 6 pixel background aperture. From inspection of the artificial point sources, we find that visual detections, however, depend almost entirely on only the several

\footnotetext{
13 http://etc.stsci.edu/etc/
} 
pixels closest to the coordinates of the source. We note that the limiting magnitudes of HST images calculated by Li et al. (2011) in their analysis of the explosion site of SN 2011fe are consistent with estimates instead made using a circular aperture enclosing $80 \%$ of the flux.

As shown in Table 1, the visual magnitude limits are comparable to the $3 \sigma$ limits estimated using the background statistics. We assign accordingly a $3 \sigma$ significance to the visual limits for the purpose of computing likelihood functions.

\subsection{Constraints on Progenitor Systems}

To convert these limits on the apparent magnitudes of a progenitor system to constraints on a stellar source, we use the Pickles (1998) library to model the spectral energy distributions (SEDs) of candidate progenitor systems of the $\mathrm{SN}$, as well as (following Li et al. 2011) blackbody spectra with temperatures of $35,000,65,000$, and $95,000 \mathrm{~K}$. For each combination of HST filter and instrument in Table 1 and all spectroscopic templates, we compute synthetic magnitudes with and without extinction from dust. After removing $E(B-V)_{\mathrm{MW}}=0.14 \mathrm{mag}$ of foreground Milky Way $\left(R_{V}=3.1\right)$ extinction, Goobar et al. (2014) estimated that additional extinction of $E(B-V)_{\mathrm{SN}}=$ $1.22 \pm 0.05$ mag with $R_{V}=1.40 \pm 0.15$ can best reproduce the shape of a premaximum optical spectrum of SN 2014J. While we adopt the Goobar et al. (2014) extinction curve for our progenitor constraints, we additionally compute synthetic magnitudes instead with an $R_{V}=3.1$ curve for M82, using the total $A_{V}$ favored by Goobar et al. (2014). As we demonstrate in Section 5, we obtain similar progenitor constraints for these differing values of $R_{V}$.

Following Li et al. (2011), we first translate the upper limit on the flux for each filtered observation to a $2 \sigma$ upper limit on the absolute magnitude $M_{V}$, using a distance modulus to M82 of $27.73 \pm 0.02 \mathrm{mag}$ (Jacobs et al. 2009). After repeating this for all observations, we identify the most constraining upper limit on $M_{V}$, and refer to this as the $2 \sigma$ " 1 -frame" limit for each spectrum template. A combined constraint is estimated next by constructing a probability function that incorporates the upper magnitude limits in all filters. The total probability (as a function of $M_{V}$ and the template spectrum) is the product of the probabilities of each filtered observation computed from the predicted model magnitude and the measured upper magnitude limit.

To calculate the combined progenitor system limit, we incrementally increase the absolute brightness $M_{V}$ until a $2 \sigma$ probability ( $p=0.954$ ) is reached. As in Li et al. (2011), the combined constraints are $\sim 0.2-0.8$ mag deeper than " 1 -frame" constraints which rely on observations in a single filter. We would expect to detect, with $95 \%$ probability, such a $2 \sigma$ source in a least one HST bandpass image, and in the coadded image in Figure 2.

Table 1 shows the detection upper limits for the coadded image of each data set, and Table 2 lists the corresponding constraint on $M_{J}$, in addition to $M_{V}$, computed for each Pickles (1998) and blackbody spectrum. For each spectrum, the photometric band providing the faintest individual absolute magnitude limit is also identified in Table 2.

In Figure 3, we plot the limits on the progenitor flux we estimate using the HST imaging. We also show measurements of the luminosity $L_{v}$ of RS Oph during its quiescent phase as a function of frequency $v$, and the uncertainty arising from current constraints on its distance.
Table 2

Stellar and Blackbody Upper Magnitude Limits

\begin{tabular}{lcccccc}
\hline \hline Star & \multicolumn{2}{c}{$M_{V}(2 \sigma)$} & & \multicolumn{2}{c}{$M_{J}(2 \sigma)$} & \multirow{2}{*}{ Most Constraining } \\
\cline { 2 - 3 } & "1-frame" & Combined & "1-frame" & Combined & "1-frame" Bandpass \\
\hline O5 V & -3.25 & -2.53 & -2.51 & -1.79 & F555W \\
B0 V & -3.25 & -2.55 & -2.55 & -1.85 & F555W \\
A0 V & -3.26 & -2.54 & -3.26 & -2.54 & F555W \\
A5 V & -3.26 & -2.48 & -3.54 & -2.76 & F555W \\
F0 V & -3.08 & -2.37 & -3.61 & -2.90 & F814W \\
F5 V & -2.94 & -2.26 & -3.76 & -3.08 & F814W \\
G0 V & -2.76 & -2.15 & -3.77 & -3.16 & F814W \\
G5 V & -2.69 & -2.08 & -3.87 & -3.26 & F814W \\
K0 V & -2.55 & -1.95 & -3.93 & -3.33 & F814W \\
K5 V & -2.04 & -1.40 & -4.21 & -3.57 & F814W \\
M0 V & -1.63 & -0.86 & -4.49 & -3.72 & F110W \\
M4 V & -0.09 & -0.57 & -4.52 & -3.86 & F110W \\
M5 V & -0.71 & -1.43 & -4.55 & -3.83 & F160W \\
B5 III & -3.25 & -2.54 & -2.92 & -2.21 & F555W \\
G0 III & -2.69 & -2.03 & -4.00 & -3.34 & F814W \\
G5 III & -2.55 & -1.90 & -4.09 & -3.44 & F814W \\
K0 III & -2.44 & -1.79 & -4.12 & -3.47 & F814W \\
K5 III & -1.81 & -1.03 & -4.48 & -3.70 & F110W \\
M0 III & -1.67 & -0.86 & -4.48 & -3.67 & F110W \\
M5 III & 0.18 & 0.90 & -4.48 & -3.76 & F160W \\
M10 III & 3.84 & 4.42 & -4.51 & -3.93 & F128N \\
B5 I & -3.26 & -2.51 & -3.09 & -2.34 & F555W \\
F0 I & -3.11 & -2.38 & -3.55 & -2.82 & F814W \\
F5 I & -3.04 & -2.33 & -3.70 & -2.99 & F814W \\
G0 I & -2.76 & -2.13 & -3.80 & -3.17 & F814W \\
G5 I & -2.60 & -1.99 & -3.91 & -3.30 & F814W \\
M2 I & -1.10 & -0.42 & & 4.40 & -3.72 & F814W \\
BB1 & -3.25 & -2.53 & & -2.62 & -1.90 & F555W \\
BB2 & -3.25 & -2.53 & & -2.50 & -1.78 & F555W \\
BB3 & -3.25 & 2.53 & & -2.45 & -1.73 & F555W \\
\hline
\end{tabular}

Notes. Limiting magnitudes in $V$ and $J$ bands in the Vega system for a point source at the explosion site. The BB1, BB2, and BB3 blackbody spectra have $35,000,65,000$, and 95,000 K temperatures, respectively. Stellar classifications are those of the Pickles (1998) spectra used as models of the potential companion. The bandpass in right column is the most constraining observation for the "1-frame" upper magnitude limits.

\subsection{RS Oph SED Model}

The luminosity of RS Oph is comparable to our upper detection limit $\left(R_{V}^{\mathrm{M} 82}=1.4 ; A_{V}^{\mathrm{M} 82}=1.7 \mathrm{mag}\right)$, and the bright white dwarf primary in RS Oph contributes significantly in bluer optical bandpasses, unlike the primary of $\mathrm{T} \mathrm{CrB}$. Therefore, we create a near-UV to near-IR model of the spectrum of RS Oph to account for the contribution of the white dwarf, and to show clearly the measurements that contribute to our constraints.

Anupama \& Mikołajewska (1999) performed a decomposition of an optical spectrum of RS Oph in its quiescent phase and find an A2 to A4 stellar spectrum for the hot component, and an M0 to M2 III spectrum for the giant companion. We fit for the linear combination of the corresponding Pickles (1998) stellar spectra that best matches the broadband magnitudes of RS Oph during quiescence. Li et al. (2011) use an effective temperature of 3750 to $3650 \mathrm{~K}$ for the giant companion typical of M0 to M2 giant stars (Straizys \& Kuriliene 1981) in their SN $2011 \mathrm{fe}$ progenitor analysis.

Evans et al. (1988) compiled $J$-, $H$-, and $K$-band magnitudes of RS Oph obtained during quiescence from 1971 through 1982 by Swings \& Allen (1972), Feast \& Glass (1974), Szkody (1977), Sherrington \& Jameson (1983), and Kenyon \& Gallagher (1983). These measurements show modest variation of \pm 0.2 


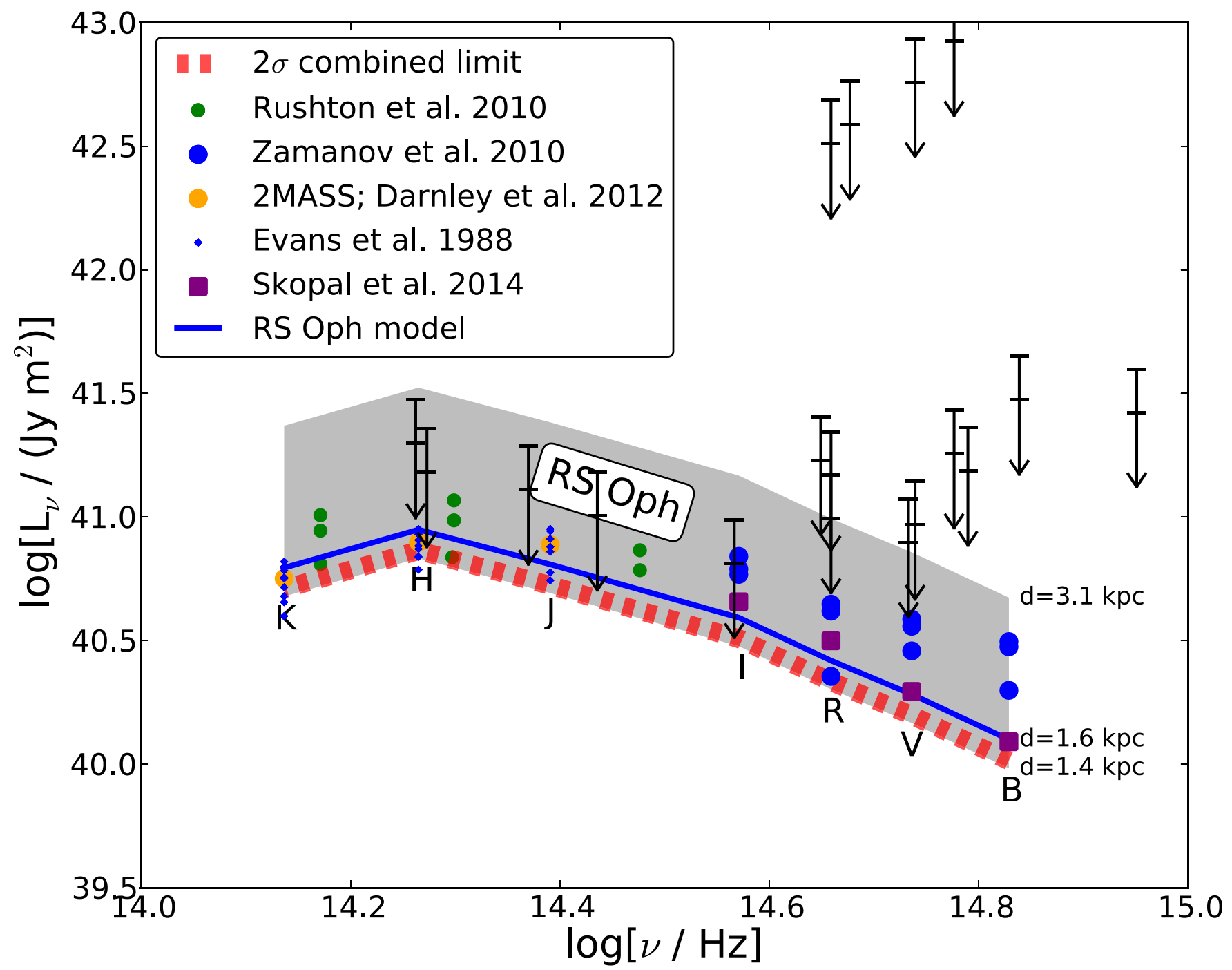

Figure 3. Constraints on the luminosity $L_{v}$ of the progenitor system against the central frequency $v$ of the bandpass filter. Values for the Galactic symbiotic nova RS Oph, a candidate single-degenerate progenitor system, in the quiescent phase are plotted for comparison. For each $H S T$ imaging data set, the arrow tip marks the estimated $1 \sigma$ upper luminosity limit, while the horizontal hatch marks along the arrow show the $2 \sigma$ and $3 \sigma$ upper limits. The thick blue line shows the conservative values we adopt as a model for the luminosity of RS Oph in the quiescent phase (with $d=1.6 \mathrm{kpc}$ ), while the gray region shows the range in $L_{v}$ for the range of possible distances to RS Oph $(1.4 \mathrm{kpc} \leqslant d \leqslant 3.4 \mathrm{kpc})$. The red dashed line represents the $2 \sigma$ limits inferred from $H S T$ observations in all bandpasses using the model SED for RS Oph. The progenitor luminosity limits shown are computed using the extinction and reddening $\left(R_{V}^{\mathrm{M} 82}=1.4 ; A_{V}^{\mathrm{M} 82}=1.7 \mathrm{mag}\right)$ values along the line of sight to SN 2014J estimated by Goobar et al. (2014). We plot the central frequencies of the Johnson-Cousins $B V R I$ and Bessell $J H K$ filters for reference. Measurements of RS Oph from Evans et al. (1988), Rushton et al. (2010), Zamanov et al. (2010), Darnley et al. (2012), and Skopal (2014) are converted to $L_{v}$ for $d=1.6 \mathrm{kpc}$.

mag, and we use the median values $(J \approx 7.67, H \approx 6.92$, $K \approx 6.62 \mathrm{mag}$ ) as representative values during the quiescent phase. Magnitudes of RS Oph measured by Two Micron All Sky Survey (Skrutskie et al. 2006) in $1999(J=7.63 \pm 0.02$, $H=6.85 \pm 0.04, K=6.50 \pm 0.01 \mathrm{mag}$; see also Darnley et al. 2012), as well as values synthesized from a near-IR spectrum (Rushton et al. 2010; Skopal 2014), are consistent with the magnitudes in quiescence assembled by Evans et al. (1988). We use the optical BVRI photometry of RS Oph during quiescence measured by Skopal (2014) to construct our model. The Skopal (2014) optical measurements are, on average, fainter than those presented by Zamanov et al. (2010) for several epochs. While Schaefer (2010) combine optical and IR colors of RS Oph taken on two dates to infer a possibly fainter flux in the IR, the combined values they present do not appear to be representative of the system in quiescence.
To estimate the luminosity of RS Oph, we use $E(B-V)=$ $0.73 \mathrm{mag}$ (Snijders 1987), which is consistent with the value derived by Anupama $\&$ Mikołajewska (1999), for an $R_{V}=3.1$ Cardelli et al. (1989) Milky Way extinction curve. Estimates for the distance to RS Oph include $\sim 1.4 \mathrm{kpc}$ from modeling of the 2006 outburst (e.g., Rupen et al. 2008), $\sim 1.6 \mathrm{kpc}$ from radio observations of expanding material in the 1985 (Hjellming et al. 1986) and 2006 (Sokoloski et al. 2006) outbursts, and $\sim 3.1 \mathrm{kpc}$ based on the assumption that the secondary fills the entire Roche lobe (Livio et al. 1986; Barry et al. 2008).

With a $V$-band total magnitude of 11.5 in quiescence, we estimate a range in absolute magnitude of $-1.49 \leqslant M_{V} \leqslant$ -3.22 for distances of 1.4-3.1 kpc. Using our decomposition of the spectrum of RS Oph in quiescence, we estimate that the giant star should be $\sim 0.65$ mag fainter in $V$ than the total magnitude of the binary system. 


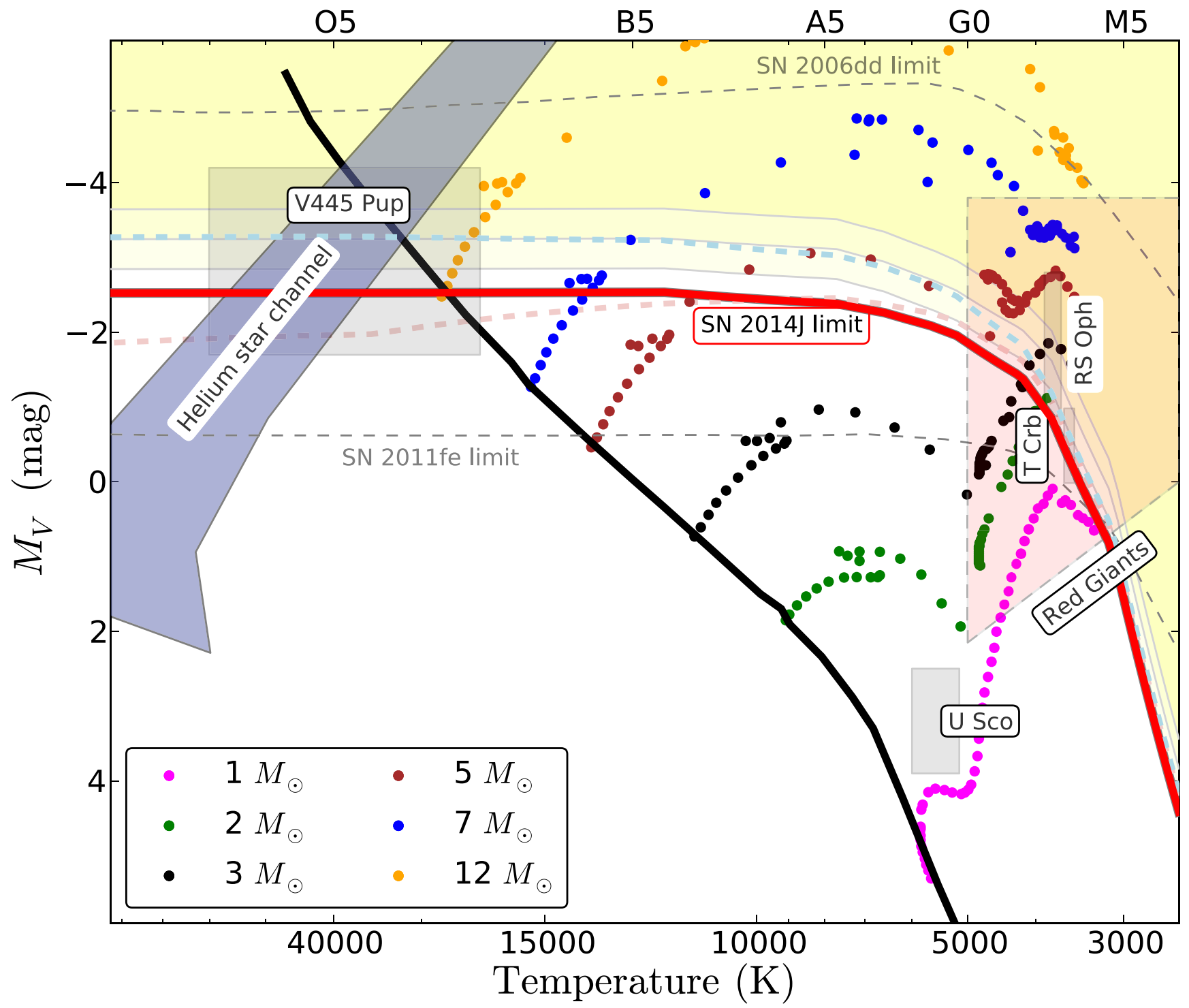

Figure 4. Constraints on the position of the SN 2014J progenitor system in the Hertzsprung-Russell (H-R) diagram. The thick bright red line corresponds to the $2 \sigma M_{V}$ limits $\left(R_{V}^{\mathrm{M} 82}=1.4 ; A_{V}^{\mathrm{M} 82}=1.7 \mathrm{mag}\right)$ that we place on the progenitor system as a function of temperature and stellar type from the combination of all limits in all HST bands. The middle solid gray line shows the $2 \sigma$ limits on the progenitor absolute magnitude obtained using the most constraining single observation, while the upper and lower adjacent gray lines provide an estimate of the uncertainty on this limit. The region brighter than the most constraining single observation limit is shaded in yellow. Starburst regions of M82 have approximately solar metallicity (Förster Schreiber et al. 2001), and we plot stellar evolutionary tracks off of the main sequence (solid black line) calculated by Lejeune \& Schaerer (2001) with appropriate abundance $(Z=0.02)$. Our upper limits likely exclude recurrent novae with luminosities comparable to that of RS Oph (Hachisu \& Kato 2001). Candidate systems U Sco (Thoroughgood et al. 2001; Hachisu et al. 1999) cannot be excluded, while T CrB (Hachisu \& Kato 2001) and V445 Pup (Woudt et al. 2009) span the upper limit. The light-red shaded region corresponds approximately to stars considered red giants and red bright giants. Dashed gray lines show the limits measured for SN 2011fe in M101 (Li et al. 2011) as well as SN 2006dd in NGC 1316 (Maoz \& Mannucci 2008) (representative of constraints for other nearby SNe Ia). The dashed pale red line shows the $2 \sigma M_{V}$ limits for $R_{V}^{\mathrm{M} 82}=3.1 \mathrm{mag}$ and $A_{V}^{\mathrm{M} 82}=1.7 \mathrm{mag}$, while the dashed pale blue line shows those for $R_{V}^{\mathrm{M} 82}=2 \mathrm{mag}$ and $A_{V}^{\mathrm{M} 82}=2.5 \mathrm{mag}$. Absolute magnitudes are computed using a distance modulus of $27.73 \pm 0.02 \mathrm{mag}$ (Jacobs et al. 2009).

\section{RESULTS}

\subsection{Progenitor Surroundings}

As can be seen in Figure 2, the SN 2014J coordinates that the $\mathrm{AO}$ analysis favors are at a greater angular distance from the nearest resolved point source than the Tendulkar et al. (2014) position. This suggests that the star at a smallest angular offset is not a mass donor to the white dwarf progenitor of SN 2014J. Our coordinates also position SN 2014J closer to the center of an apparent dust cloud whose silhouette is visible in optical HST images.

\subsection{Progenitor Models}

Figure 4 shows the derived constraints on the position of the progenitor system of SN 2014J in the Hertzsprung-Russell (H-R) diagram. The upper luminosity limit excludes the bright extent of the region, marked in pale red, occupied by red giant stars. The faint boundary of the red giant region corresponds approximately to the least luminous red giants in the Hipparcos catalog (Perryman et al. 1997), and we truncate the red giant region at an upper effective temperature of $5000 \mathrm{~K}$. Stars more luminous than the plotted $M_{V} \approx 3.8$ mag bright boundary may instead be classified as red supergiant stars. 
With the exception of values for RS Oph (see Section 4.3), we plot the ranges of effective temperature and $M_{V}$ values collected by Li et al. (2011) for Galactic candidate systems. Our luminosity limits are comparable to our faintest estimates for the luminosity of RS Oph in quiescence, including the uncertainty in its distance in the Galaxy. The upper $M_{V}$ line intersects the $M_{V}-T$ area corresponding to the less luminous Galactic symbiotic system T CrB (Hachisu \& Kato 2001).

$\mathrm{U}$ Sco is a recurrent nova and supersoft X-ray source with a large white dwarf mass of $1.55 \pm 0.24 M_{\odot}$ (Thoroughgood et al. 2001) and a subgiant companion. This candidate progenitor system is substantially too faint for detection in our archival images, as was the case for SN $2011 \mathrm{fe}$.

A candidate He-rich single-degenerate progenitor system is the He nova V445 Puppis (V445 Pup). We use the Woudt et al. (2009) estimate for $M_{V}$ based on a parallax distance measurement of $8.2 \pm 0.5 \mathrm{kpc}$ from the expansion of a bipolar shell after an eruption in year 2000. The upper luminosity limits for SN 2014J extend across the region of the H-R diagram corresponding to V445 Pup. Figure 4 shows the theoretical limits on the He channel computed by Li et al. (2011) using Liu et al. (2010) models and bolometric corrections from Torres (2010). Our measured limits are fainter than the predicted luminosities of He-star-channel progenitors with comparatively cool temperatures $(T \lesssim 35,000 \mathrm{~K})$.

For comparison, we plot the luminosity limits for the progenitors of SN 2011fe (Li et al. 2011) and SN 2006dd (Maoz \& Mannucci 2008); the latter provides a constraint typical of SNe Ia other than SN 2011fe. For companions with very low effective temperature $(T<3000 \mathrm{~K})$, limits on the progenitor SN 2014J are significantly fainter than those for SN $2011 \mathrm{fe}$, because the SN 2014J archival images extend to the near-IR.

Now referring to the stellar evolutionary tracks off the main sequence computed by Lejeune \& Schaerer (2001), we find that a mass donor with effective temperature cooler than $4000 \mathrm{~K}$ would need to have a mass less than $2 M_{\odot}$. The plotted evolutionary tracks are models with abundance $(Z=0.02)$ consistent with the metallicity of M82 starburst regions (Förster Schreiber et al. 2001). The derived luminosity limits are significantly brighter than a hypothetical binary system of two white dwarfs that does not experience a long-lived merger phase (Shen et al. 2012), or a white-dwarf/main-sequence binary (e.g., Wheeler 2012).

Comparison among the bright red $\left(R_{V}^{\mathrm{M} 82}=3.1 \mathrm{mag}\right.$; $A_{V}^{\mathrm{M} 82}=1.7 \mathrm{mag}$; Goobar et al. 2014), the dashed pale red $\left(R_{V}^{\mathrm{M} 82}=3.1 \mathrm{mag} ; A_{V}^{\mathrm{M} 82}=1.7 \mathrm{mag}\right)$, and the dashed pale blue $\left(R_{V}^{\mathrm{M} 82}=2 \mathrm{mag} ; A_{V}^{\mathrm{M} 82}=2.5 \mathrm{mag}\right)$ lines shows that the progenitor constraints exhibit only modest change within the range of dust parameters reported from analyses of the SN 2014J light curve and spectra (e.g., Goobar et al. 2014; Patat et al. 2014).

While the total integration times of pre-explosion HST broadband imaging of the site of SN 2014J are almost all greater than that of the site of SN $2011 \mathrm{fe}$, and M82 $(d \approx 3.5$; Jacobs et al. $2009)$ is at a smaller distance than M101 ( $d \approx 6.4 \mathrm{Mpc}$; Shappee \& Stanek 2011), the upper flux limits at optical wavelengths that we estimate for the progenitor of SN 2014J are less constraining than those for the progenitor of SN 2011fe (Li et al. 2011). The brighter optical constraints on the progenitor of SN 2014J arise from the high extinction inferred along the line of sight to SN 2014J, and from the bright and varying local background in M82. In contrast, the spectra and colors of SN 2011fe were consistent with a lack of extinction and it occurred at a location with low surface brightness. In the ACS WFC F814W FLT images of the explosion sites, we measure an average of $\sim 0.13$ counts $\mathrm{s}^{-1}$ pixel $^{-1}$ close to the explosion site of $\mathrm{SN}$ 2011fe in M101, and $~ 5.3$ counts $\mathrm{s}^{-1}$ pixel $^{-1}$ close to Type Ia SN 2014J in M82.

\section{CONCLUSIONS}

We have used archival, pre-explosion HST images of M82 in the near-UV through near-IR to place constraints on the progenitor system of the SN Ia 2014J. Assuming that the extinction and selective extinction along the line of sight to the SN estimated from the SN light curve and optical spectra are approximately correct (e.g., Goobar et al. 2014; Patat et al. 2014), we can exclude a progenitor system with a bright red giant mass-donor companion, including recurrent novae with luminosities comparable to the Galactic prototype symbiotic system RS Oph. Our limits are fainter than the predicted luminosity of He-starchannel progenitors with comparatively low effective temperature. The available near-IR M82 data provide a fainter limit for mass donors with very low effective temperatures $(T<3000 \mathrm{~K})$ than was possible at the explosion site of SN $2011 \mathrm{fe}$ in M101. A hypothetical progenitor system consisting of two white dwarf stars that does not experience a long-lived merger phase (Shen et al. 2012) would have a luminosity significantly fainter than the upper limits we estimate.

We greatly appreciate the critical contribution of observing time from Vithal Tilvi, Nicola Mehrtens, Casey Papovich, and Mark Dickinson that enabled us to complete the Keck AO imaging, as well as their encouragement and helpful suggestions on the manuscript. We thank Michael Liu, Shriharsh Tendulkar, Yi Cao, and Trent Dupuy for generously providing details of their NIRC2 AO observations to aid our team in deciding whether any further imaging could be helpful. Correspondence with both Matthew Darnley and G. C. Anupama was also useful. A.V.F.'s group at UC Berkeley has received generous financial assistance from the Christopher R. Redlich Fund, the TABASGO Foundation, Gary and Cynthia Bengier, and NSF grant AST-1211916. This work was also supported by NASA grants AR-12623 and AR-12850 from the Space Telescope Science Institute, which is operated by AURA, Inc., under NASA contract NAS 5-26555. K.J.S. is supported by NASA through Einstein Postdoctoral Fellowship grant number PF1120088 awarded by the Chandra X-ray Center, which is operated by the Smithsonian Astrophysical Observatory for NASA under contract NAS8-03060.

Some of the data presented herein were obtained at the W. M. Keck Observatory from telescope time allocated to NASA through the agency's scientific partnership with the California Institute of Technology and the University of California. The Observatory was made possible by the generous financial support of the W. M. Keck Foundation. We recognize the Hawaiian community for the opportunity to conduct these observations from the summit of Mauna Kea.

\section{REFERENCES}

Anupama, G. C., \& Mikołajewska, J. 1999, A\&A, 344, 177

Barry, R. K., Mukai, K., Sokoloski, J. L., et al. 2008, in ASP Conf. Ser. 401, RS Ophiuchi (2006) and the Recurrent Nova Phenomenon, ed. A. Evans, M. F. Bode, T. J. O’Brien, \& M. J. Darnley (San Francisco, CA: ASP), 52 Bertin, E., \& Arnouts, S. 1996, A\&AS, 117, 393

Cardelli, J. A., Clayton, G. C., \& Mathis, J. S. 1989, ApJ, 345, 245

Chandler, C. J., \& Marvil, J. 2014, ATel, 5812, 1

Childress, M., Aldering, G., Antilogus, P., et al. 2013, ApJ, 770, 108

Darnley, M. J., Ribeiro, V. A. R. M., Bode, M. F., Hounsell, R. A., \& Williams, R. P. 2012, ApJ, 746, 61 
Evans, A., Callus, C. M., Albinson, J. S., et al. 1988, MNRAS, 234, 755 Feast, M. W., \& Glass, I. S. 1974, MNRAS, 167, 81

Förster Schreiber, N. M., Genzel, R., Lutz, D., Kunze, D., \& Sternberg, A. 2001, ApJ, 552, 544

Fossey, J., Cooke, B., Pollack, G., Wilde, M., \& Wright, T. 2014, CBET, 3792, 1

Geier, S., Marsh, T. R., Wang, B., et al. 2013, A\&A, 554, A54

Goobar, A., Johansson, J., Amanullah, R., et al. 2014, ApJL, 784, L12

Hachisu, I., \& Kato, M. 2001, ApJ, 558, 323

Hachisu, I., Kato, M., Nomoto, K., \& Umeda, H. 1999, ApJ, 519, 314

Han, Z., \& Podsiadlowski, P. 2004, MNRAS, 350, 1301

Hillebrandt, W., \& Niemeyer, J. C. 2000, ARA\&A, 38, 191

Hjellming, R. M., van Gorkom, J. H., Taylor, A. R., et al. 1986, ApJL, 305, L71

Iben, I., Jr., \& Tutukov, A. V. 1984, ApJS, 54, 335

Jacobs, B. A., Rizzi, L., Tully, R. B., et al. 2009, AJ, 138, 332

Kasen, D., \& Plewa, T. 2005, ApJL, 622, L41

Kasen, D., Röpke, F. K., \& Woosley, S. E. 2009, Natur, 460, 869

Kasen, D., \& Woosley, S. E. 2007, ApJ, 656, 661

Kelly, P. L., Hicken, M., Burke, D. L., Mandel, K. S., \& Kirshner, R. P. 2010, ApJ, 715,743

Kelly, P. L., Kirshner, R. P., \& Pahre, M. 2008, ApJ, 687, 1201

Kenyon, S. J., \& Gallagher, J. S. 1983, AJ, 88, 666

Lampeitl, H., Smith, M., Nichol, R. C., et al. 2010, ApJ, 722, 566

Lejeune, T., \& Schaerer, D. 2001, A\&A, 366, 538

Li, W., Bloom, J. S., Podsiadlowski, P., et al. 2011, Natur, 480, 348

Liu, W.-M., Chen, W.-C., Wang, B., \& Han, Z. W. 2010, A\&A, 523, A3

Livio, M., Truran, J. W., \& Webbink, R. F. 1986, ApJ, 308, 736

Maoz, D., \& Mannucci, F. 2008, MNRAS, 388, 421

Mazzali, P. A., Röpke, F. K., Benetti, S., \& Hillebrandt, W. 2007, Sci, 315,825

Munari, U., \& Renzini, A. 1992, ApJL, 397, L87

Nelemans, G., Voss, R., Roelofs, G., \& Bassa, C. 2008, MNRAS, 388, 487

Nielsen, M. T. B., Gilfanov, M., Bogdan, A., Woods, T. E., \& Nelemans, G. 2014, arXiv:1402.2896

Nomoto, K. 1982, ApJ, 253, 798

Patat, F., Chugai, N. N., Podsiadlowski, P., et al. 2011, A\&A, 530, A63

Patat, F., Taubenberger, S., Baade, D., et al. 2014, ATel, 5830, 1

Perlmutter, S., Aldering, G., Goldhaber, G., et al. 1999, ApJ, 517, 565
Perryman, M. A. C., Lindegren, L., Kovalevsky, J., et al. 1997, A\&A, 323, L49 Phillips, M. M. 1993, ApJL, 413, L105

Pickles, A. J. 1998, PASP, 110, 863

Raskin, C., Scannapieco, E., Rhoads, J., \& Della Valle, M. 2009, ApJ, 707, 74

Riess, A. G., Filippenko, A. V., Challis, P., et al. 1998, AJ, 116, 1009

Riess, A. G., Press, W. H., \& Kirshner, R. P. 1996, ApJ, 473, 88

Rupen, M. P., Mioduszewski, A. J., \& Sokoloski, J. L. 2008, ApJ, 688, 559

Rushton, M. T., Kaminsky, B., Lynch, D. K., et al. 2010, MNRAS, 401, 99

Schaefer, B. E. 2010, ApJS, 187, 275

Schlafly, E. F., \& Finkbeiner, D. P. 2011, ApJ, 737, 103

Shappee, B. J., \& Stanek, K. Z. 2011, ApJ, 733, 124

Shen, K. J., \& Bildsten, L. 2014, ApJ, 785, 61

Shen, K. J., Bildsten, L., Kasen, D., \& Quataert, E. 2012, ApJ, 748, 35

Sherrington, M. R., \& Jameson, R. F. 1983, MNRAS, 205, 265

Skopal, A. 2014, NewA, in press (arXiv:1402.6126)

Skrutskie, M. F., Cutri, R. M., Stiening, R., et al. 2006, AJ, 131, 1163

Snijders, M. A. J. 1987, Ap\&SS, 130, 243

Sokoloski, J. L., Luna, G. J. M., Mukai, K., \& Kenyon, S. J. 2006, Natur, 442,276

Straizys, V., \& Kuriliene, G. 1981, Ap\&SS, 80, 353

Sullivan, M., Conley, A., Howell, D. A., et al. 2010, MNRAS, 406, 782

Swings, J. P., \& Allen, D. A. 1972, PASP, 84, 523

Szkody, P. 1977, ApJ, 217, 140

Tendulkar, S. P., Liu, M. C., Dupuy, T. J., \& Ca, Y. 2014, ATel, 5789, 1

Thoroughgood, T. D., Dhillon, V. S., Littlefair, S. P., Marsh, T. R., \& Smith, D. A. 2001, MNRAS, 327, 1323

Torres, G. 2010, AJ, 140, 1158

van den Heuvel, E. P. J., Bhattacharya, D., Nomoto, K., \& Rappaport, S. A. 1992, A\&A, 262, 97

Wang, B., Meng, X., Chen, X., \& Han, Z. 2009, MNRAS, 395, 847

Webbink, R. F. 1984, ApJ, 277, 355

Wheeler, J. C. 2012, ApJ, 758, 123

Whelan, J., \& Iben, I. J. 1973, ApJ, 186, 1007

Wizinowich, P. L., Le Mignant, D., Bouchez, A. H., et al. 2006, PASP, 118, 297

Woudt, P. A., Steeghs, D., Karovska, M., et al. 2009, ApJ, 706, 738

Yoon, S.-C., \& Langer, N. 2003, A\&A, 412, L53

Zamanov, R. K., Boeva, S., Bachev, R., et al. 2010, MNRAS, 404, 381

Zheng, W., Shivvers, I., Filippenko, A. V., et al. 2014, ApJL, 783, L24 